\title{
A!
}

This is an electronic reprint of the original article.

This reprint may differ from the original in pagination and typographic detail.

Virkkala, V.; Havu, V.; Tuomisto, F.; Puska, M.J.

\section{Native Point Defect Energetics in GaSb: enabling p-type conductivity of undoped GaSb}

Published in:

Physical Review B

DOI:

10.1103/PhysRevB.86.144101

Published: 01/10/2012

Document Version

Publisher's PDF, also known as Version of record

Please cite the original version:

Virkkala, V., Havu, V., Tuomisto, F., \& Puska, M. J. (2012). Native Point Defect Energetics in GaSb: enabling ptype conductivity of undoped GaSb. Physical Review B, 86(14), 1-7. [144101].

https://doi.org/10.1103/PhysRevB.86.144101

This material is protected by copyright and other intellectual property rights, and duplication or sale of all or part of any of the repository collections is not permitted, except that material may be duplicated by you for your research use or educational purposes in electronic or print form. You must obtain permission for any other use. Electronic or print copies may not be offered, whether for sale or otherwise to anyone who is not an authorised user. 


\title{
Native point defect energetics in GaSb: Enabling $p$-type conductivity of undoped GaSb
}

\author{
Ville Virkkala, Ville Havu, Filip Tuomisto, and Martti J. Puska \\ COMP Department of Applied Physics, Aalto University, P.O. Box 11100, FIN-00076 Aalto, Finland
}

(Received 8 May 2012; revised manuscript received 28 August 2012; published 1 October 2012)

\begin{abstract}
The energetics of native point defects in GaSb is studied using the density-functional theory within the hybrid functional scheme (HSE06). Our results indicate that the $\mathrm{Ga}_{\mathrm{Sb}}$ antisite has the lowest formation energy and could thus be the acceptor defect responsible for the $p$-type conductivity of undoped $\mathrm{GaSb}$. We find also that the $\mathrm{Sb}_{\mathrm{Ga}}$ antisite has a remarkably low formation energy in Sb-rich growth conditions and it should act as a donor for all Fermi level positions in the band gap. However, we suggest that the structural metastability of the $\mathrm{Sb}_{\mathrm{Ga}}$ antisite or extrinsic point defects, namely carbon and in particular oxygen, may neutralize its compensating character.
\end{abstract}

DOI: 10.1103/PhysRevB.86.144101

PACS number(s): 61.72.J-, 71.55.Eq, 71.15.Nc

\section{INTRODUCTION}

Native defects and unintentional impurities introduced during the growth of compound semiconductors often cause the material to turn to a $p$ or $n$-type. Via compensation, these defects may also hinder crucially the doping of the semiconductor materials. Specially GaSb, which is an important material from both material and device points of views, is always $p$-type when undoped. ${ }^{1}$ The technological importance of $\mathrm{GaSb}$ stems from the fact that it can be used as a substrate material for other ternary and quaternary III-V compounds whose band gaps cover a wide spectral range from 0.8 to $4.3 \mu \mathrm{m}$ (Ref. 1). GaSb also shows a lot of potential as the optically active material in various optoelectronic devices ${ }^{1}$ and recently it has attained a lot of attention in vertical-externalcavity surface emitting lasers. ${ }^{2}$ The usefulness of GaSb is enhanced by the fact that its direct band gap can be significantly narrowed by replacing $\mathrm{Sb}$ with just a small fraction (around $1 \%$ ) of nitrogen. 3,4

The origin of the $p$-type conductivity of the undoped $\mathrm{GaSb}$ has been debated. It is often connected to $\mathrm{Ga}$ antisites or vacancies. ${ }^{1}$ For example, based on positron lifetime studies, Ling et al. ${ }^{5}$ concluded that most likely the existence of Gasb antisites resulted in the $p$-type behavior whereas Ga vacancies could not be the cause. This identification was motivated also by the results of the density-functional theory (DFT) electronic structure calculations by Hakala et al. ${ }^{6}$ which showed that $\mathrm{Ga}_{\mathrm{Sb}}$ acts clearly as the most important acceptor from the Ga-rich growth conditions far to the Sb-rich stoichiometry. On the other hand, Peles et al. ${ }^{7}$ suggested on the basis of DFT calculations that interstitial hydrogen would act as a shallow acceptor in $\mathrm{GaSb}$.

Native point defects in GaSb have been studied extensively by Hakala et al. ${ }^{6}$ within the DFT framework. They used the local density approximation (LDA), norm-conserving pseudopotentials, and the plane-wave expansion for electron wave functions and densities. The Gasb antisite and the $\mathrm{Ga}$ interstitials were found as the most important acceptorand donor-type defects, respectively. The abundances of the acceptor-type Ga vacancy and the electrically nonactive (existing only in the neutral charge state) $\mathrm{Sb}_{\mathrm{Ga}}$ antisite were predicted as significant only for very $\mathrm{Sb}$-rich growth conditions.

Despite the high expectations for the usage of $\mathrm{GaSb}$ it has been the subject of a relatively small number of computational studies in comparison with other III-V compounds, especially GaAs. This motivates us to revisit the defect problematics of $\mathrm{GaSb}$, especially in terms of the source of the $p$-type conductivity in $\mathrm{GaSb}$ with the help of state-of-the-art computational methods. In contrast to the earlier DFT approaches used, the state-of-the-art methods can tackle the problem of the underestimation of the band gap in the (semi)local approximations for the electron exchange and correlation and they correct more accurately for the spurious electrostatic defect-defect interactions in the supercell approximation. Both of the problems can affect the accurate calculation of the defect ionization level positions as well as the defect formation energies. ${ }^{8}$ More specifically, we use a hybrid exchange-correlation functional which substitutes a portion of the semilocal exchange by the nonlocal Hartree-Fock (HF) exchange. The hybrid functionals allow one to adjust the band gap to the experimental value giving a straightforward scheme to predict the energetics of the different charge states for different Fermi level positions within the band gap. To overcome the finite-size effects due to the periodic repetition of a charged supercell we use the method by Freysoldt et al.. ${ }^{9}$ The reliable treatment of the finite-size effects is especially important when using the hybrid functionals because, at the moment, their computational expense does not allow the use of large supercells of more than 200 atoms. Recently, Komsa and Pasquarello ${ }^{10}$ assessed the accuracy of hybrid functionals with a finite-size correction approach in the case of the $\mathrm{As}_{\mathrm{Ga}}$ antisite in GaAs. They found that the hybrid functionals tuned to give the experimental band gap reproduce the experimental ionization level positions within $\sim 0.2 \mathrm{eV}$. This gives credence to our application of these methods in the case of native defects in $\mathrm{GaSb}$.

In the present work, we found, in agreement with the results by Hakala et al., ${ }^{6}$ that $\mathrm{Ga}_{\mathrm{Sb}}$ antisites are energetically the most favored acceptor defects, especially for Ga-rich growth conditions. However, in sharp contrast, we find that $\mathrm{Sb}_{\mathrm{Ga}}$ antisites exhibit donor states $2+$ and $1+$ in the band gap and that consequently their formation energies in $p$-type materials are low. The existence of the $\mathrm{Sb}_{\mathrm{Ga}}$ donor levels is in line with the (computational) findings for anion antisite defects in other III-V compound semiconductors, notably in GaAs (Refs. 10 and 11) but very interestingly also in AlSb (Ref. 12). The prediction of the $\mathrm{Sb}_{\mathrm{Ga}}$ donor levels associated with low defect formation energies is a challenge for explaining the 
origin of the $p$-type conductivity of $\mathrm{GaSb}$. We found that $\mathrm{Sb}_{\mathrm{Ga}}$ exhibits also a metastable $C_{3 v}$-symmetry configuration which is similar to the EL2 defect in GaAs (see, e.g., Ref. 11) and is also predicted to exists in other III-V compounds [e.g., in $\mathrm{AlSb}$ (Ref. 12)]. The $C_{3 v}$ configuration is neutral for the whole range of the Fermi level in the band gap. Below we discuss the transition of the $\mathrm{Sb}_{\mathrm{Ga}}$ antisite to this electrically inactive state.

Even though our main focus is on the native point defects, we also perform a very limited study of extrinsic point defects, namely carbon at the substitutional Sb site and oxygen at the interstitial cation site as possible sources of $p$-type conductivity. Erhart et al. ${ }^{13}$ studied these defects in AlSb and found them as the most detrimental impurities that can be unintentionally incorporated during the growth process. They used the LDA functional and 64-atom supercell to study various extrinsic point defects in $\mathrm{AlSb}$ and found that substitutional carbon at the $\mathrm{Sb}$ site acts as a singly acceptor for the whole range of the Fermi level. Oxygen at the interstitial cation site was shown to act as a single or double acceptor with a notably low formation energy. In this paper we show that oxygen and carbon act also as acceptors in $\mathrm{GaSb}$ and could thus compensate the donor character of the $\mathrm{Sb}_{\mathrm{Ga}}$ antisite.

The organization of the present paper is as follows. The computational methods with parameters chosen and benchmark results obtained are given in Sec. II. Section III presents the energetics and geometries of the different native defects in $\mathrm{GaSb}$ and those of the carbon and oxygen impurities. The implications of the results are discussed in Sec. IV and Sec. V is a short summary.

\section{METHODS}

All the present calculations are performed using the Vienna $a b$ initio simulation package (VASP) ${ }^{14}$ with the projector augmented wave (PAW) ${ }^{15}$ method. The HSE06 hybrid functional ${ }^{16}$ is used except in some test and reference calculations where the LDA functional is employed. For the default HF mixing constant of $25 \%$ the obtained lattice constant is $6.16 \AA$ and the band gap is $0.64 \mathrm{eV}$. However, we adjust the mixing constant to reproduce the experimental band gap of $0.81 \mathrm{eV}$ at $0 \mathrm{~K}$. This is a commonly recommended procedure when hybrid functionals are used in defect calculations. ${ }^{10,17}$ The adjusted HF mixing constant is $29 \%$ and the corresponding lattice constant $6.15 \AA$, which is somewhat larger than the experimental value of $6.10 \AA$ (Ref. 18.) We use the plane-wave cutoff energy of $300 \mathrm{eV}$ which provides a good convergence of the total energy. For the HSE range separation parameter $\mu$ we use the value $0.2 / \AA$. All the calculations are performed in a 64-atom simple cubic supercell and the $2 \times 2 \times 2$ MonkhorstPack set is used for the k-point sampling in most of the cases. The few exceptions are explained in more detail below. Atoms are relaxed to their ground-state positions without any symmetry restrictions. The stopping criterion for ionic relaxation is that the total force acting on each atom is less than $0.02 \mathrm{eV} / \AA$ in magnitude. All the cases are calculated as spin polarized.

The studied systems are $\mathrm{Ga}\left(V_{\mathrm{Ga}}\right)$ and $\mathrm{Sb}\left(V_{\mathrm{Sb}}\right)$ vacancies, $\mathrm{Ga}$ and $\mathrm{Sb}$ interstitials at the hexagonal $(H)$ site and at the tetrahedral sites $\left(T_{\mathrm{c}}, T_{\mathrm{a}}\right)$, and $\mathrm{Ga}\left(\mathrm{Ga}_{\mathrm{Sb}}\right)$ and $\mathrm{Sb}\left(\mathrm{Sb}_{\mathrm{Ga}}\right)$ antisites. At the $T_{\mathrm{a}}$ interstitial site the defect has four anions and at the
$T_{\mathrm{c}}$ interstitial site four cations as nearest neighbors. We also studied two $\mathrm{Sb}$ atoms sharing one $\mathrm{Sb}$ site, $\mathrm{Sb}$ split-interstitial $\left[(\mathrm{Sb}-\mathrm{Sb})_{\mathrm{Sb}\langle 110\rangle}\right]$, oriented along the $\langle 110\rangle$ direction, which is reported to have a rather low formation energy in $\mathrm{AlSb}$ (Ref. 19). The extrinsic point defects studied in this work are carbon at the substitutional $\mathrm{Sb}$ site $\left(\mathrm{C}_{\mathrm{Sb}}\right)$ and oxygen at the interstitial cation site $\left[\mathrm{O}\left(T_{c}\right)\right]$. These extrinsic point defects are chosen because they act as acceptors in AlSb and their formation energies were found to be the lowest ones for carbon and oxygen impurities in AlSb (Ref. 13).

To overcome the finite-size effects due to periodic repetition of the net defect charge $q$ we use the method by Freysoldt et al.. ${ }^{9}$ The defect charge distribution $q_{d}(\mathbf{r})$ is approximated by a Gaussian of $1 a_{0}$ in width and for the macroscopic highfrequency dielectric constant $\varepsilon$ of GaSb the experimental value of 14.4 (Ref. 20) is used. For unrelaxed supercells the potential alignment constant $C$, needed to align the energy levels of the defect and bulk supercells, can be determined visually. ${ }^{9}$ However, in the case of relaxed supercells this method cannot be directly used because the nuclei in the defect supercell move relative to their positions in the bulk supercell and, thus, the changes in ionic potentials completely swamp the underlying defect potential. That is why in this work $C$ is determined by calculating the potential difference between the defect and bulk supercells at a point far from the defect and subtracting the periodic long-range potential $\tilde{V}_{q}^{l r}\left(\mathbf{r}_{\text {far }}\right)$ of the defect charge distribution at this point from the difference, that is,

$$
C=\tilde{V}_{\text {defect }}\left(\mathbf{r}_{\text {far }}\right)-\tilde{V}_{\text {bulk }}\left(\mathbf{r}_{\text {far }}\right)-\tilde{V}_{q}^{l r}\left(\mathbf{r}_{\text {far }}\right) .
$$

Above, $\tilde{V}_{\text {defect }}\left(\mathbf{r}_{\text {far }}\right)$ and $\tilde{V}_{\text {bulk }}\left(\mathbf{r}_{\text {far }}\right)$ are the electrostatic potentials far from the defect in the defect and bulk supercells, respectively.

The defect formation energies for intrinsic defects are calculated using the formula ${ }^{21}$

$$
\begin{aligned}
E^{f}= & E_{\mathrm{tot}}[\mathrm{def}+\mathrm{bulk}]-E_{\mathrm{tot}}[\mathrm{bulk}]-n_{\mathrm{Ga}} \mu_{\mathrm{Ga}}-n_{\mathrm{Sb}} \mu_{\mathrm{Sb}} \\
& +q\left(E_{v}+E_{f}\right)-\Delta E
\end{aligned}
$$

where $E_{v}$ is set equal to the valence band maximum (VBM), $E_{f}$ is the Fermi level referenced to the valence band maximum, $\mu_{\mathrm{Ga}}$ and $\mu_{\mathrm{Sb}}$ are the chemical potentials of the $\mathrm{Ga}$ and $\mathrm{Sb}$ atoms, respectively. Further, $n_{\mathrm{Ga}}$ and $n_{\mathrm{Sb}}$ are the numbers of the removed (a negative number) or added (a positive number) $\mathrm{Ga}$ and $\mathrm{Sb}$ atoms, respectively, and $\Delta E$ is the energy correction term $^{9}$

$\Delta E=\frac{2 \pi}{\varepsilon \Omega} \sum_{\mathbf{G} \neq 0}^{|\mathbf{G}| \leqslant G_{\text {cut }}} \frac{q_{d}(|\mathbf{G}|)^{2}}{|\mathbf{G}|^{2}}-\frac{1}{\pi \varepsilon} \int_{0}^{G_{\mathrm{cut}}} q_{d}(g)^{2} d g+q C$.

In the above, $q_{d}(g)$ is the model charge distribution in the reciprocal space, $\Omega$ is the supercell volume, and $G_{\text {cut }}$ is an appropriately chosen cutoff radius ensuring convergence of the correction term. In $\mathrm{Ga}$ ( $\mathrm{Sb}$ )-rich growth conditions $\mu_{\mathrm{Ga}}\left(\mu_{\mathrm{Sb}}\right)$ is equal to that of $\mu_{\mathrm{Ga}[\mathrm{bulk}]}\left(\mu_{\mathrm{Sb}[\mathrm{bulk}]}\right)$ and in $\mathrm{Sb}$ (Ga)-rich growth conditions $\mu_{\mathrm{Ga}}\left(\mu_{\mathrm{Sb}}\right)$ is obtained as $\mu_{\mathrm{Ga}}=$ $\mu_{\mathrm{GaSb}}-\mu_{\mathrm{Sb}[\mathrm{bulk}]}\left(\mu_{\mathrm{Sb}}=\mu_{\mathrm{GaSb}}-\mu_{\mathrm{Ga}[\mathrm{bulk}]}\right)$, where $\mu_{\mathrm{GaSb}}$ is the energy of the two-atom unit of bulk GaSb (Ref. 21). The chemical potentials for bulk $\mathrm{Ga}$ and $\mathrm{Sb}$ are calculated in their point symmetries Cmca and $R \overline{3} \mathrm{~m}$, respectively. The comparison of the calculated enthalpy of formation for $\mathrm{GaSb}$ 
$\Delta H=\mu_{\mathrm{GaSb}}-\mu_{\mathrm{Ga}[\mathrm{bulk}]}-\mu_{\mathrm{Sb}[\mathrm{bulk}]}=-0.46 \mathrm{eV}$, with the experimental value of $-0.43 \mathrm{eV}$ (Ref. 22), serves also as a benchmark test for our DFT calculations.

In calculating the formation energies for the impurity defects we need to add the term ${ }^{13}$

$$
-\sum_{i}^{i m p} \Delta n_{i}\left(\mu_{i}^{\text {bulk }}+\Delta \mu_{i}\right)
$$

into Eq. (2), where $\Delta n_{i}$ denotes the difference in the number of impurity atoms $i$ between the bulk and defect supercells, $\mu_{i}^{\text {bulk }}$ is the chemical potential of impurity $i$ in its reference state, and $\Delta \mu_{i}$ is the change in the chemical potential of this impurity with respect to bulk chemical potential. The chemical potential $\mu_{i}^{\text {bulk }}$ is calculated in the most stable phase for the impurity (graphite for $\mathrm{C}$ and $\mathrm{O}_{2}$ for $\mathrm{O}$ ) and $\Delta \mu_{i}$ is set to zero. We also found it necessary to increase the energy cutoff radius of the plane-wave basis set to $400 \mathrm{eV}$ in the case of extrinsic point defects.

The sources of errors within the used DFT framework are related to the k-point sampling and to the finite-size effects due to limited supercell size. On the basis of the the test calculations with the $4 \times 4 \times 4$ k-point sampling we estimate the k-point sampling error for the relevant defects to be $<$ $0.1 \mathrm{eV}$. However, for the neutral $\mathrm{Sb}$ antisite in the $T_{d}$ and $C_{3 v}$ configurations and for the $\mathrm{Sb}$ vacancy in the -1 charge state we found it necessary to use the $4 \times 4 \times 4$ k-point sampling for the DFT part. The ionic relaxations were made similarly to the other cases. Using the LDA functional, we have studied the convergence of the formation energy with respect to supercell size in the case of Ga vacancies in charge states from 0 to -3 . We found that for all charge states the formation energies have converged better than $0.2 \mathrm{eV}$.

\section{RESULTS}

\section{A. Ionization levels and ionic configurations for native point defects}

Figure 1 shows the different charge states and the ensuing ionization levels for the different vacancy and antisite defects in GaSb. The level positions are based on the numerical data given in Table I. The Ga vacancy is stable in the charge sates from 0 to -3 . These charge states were found also for the Ga vacancy in GaAs in hybrid functional calculations by Komsa and Pasquarello. ${ }^{23}$ The only difference is that now the

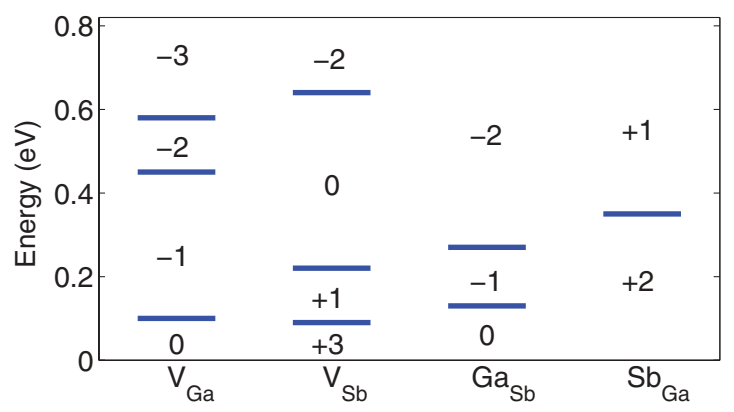

FIG. 1. (Color online) Calculated stability regions of different charge states and the ensuing ionization levels for vacancies and antisites in $\mathrm{GaSb}$.
TABLE I. Calculated formation energies of the stable vacancy, antisite, and interstitial defects in $\mathrm{GaSb}$ in $\mathrm{Ga}$-rich and $\mathrm{Sb}$-rich growth conditions corresponding to the Fermi level at the VBM.

\begin{tabular}{|c|c|c|}
\hline Defect & $E_{f}(\mathrm{eV}) \mathrm{Ga}$ rich & $E_{f}(\mathrm{eV}) \mathrm{Sb}$ rich \\
\hline$V_{\mathrm{Gg}}^{3-}$ & 4.10 & 3.63 \\
\hline$V_{\mathrm{Ga}}^{2-}$ & 3.52 & 3.05 \\
\hline$V_{\mathrm{Ga}}^{1-}$ & 3.07 & 2.60 \\
\hline$V_{\mathrm{Ga}}^{0}$ & 2.97 & 2.50 \\
\hline$V_{\mathrm{Sb}}^{2-}$ & 4.31 & 4.78 \\
\hline$V_{\mathrm{Sb}}^{0}$ & 3.03 & 3.50 \\
\hline$V_{\mathrm{Sb}}^{1+}$ & 2.81 & 3.27 \\
\hline$V_{\mathrm{Sb}}^{3+}$ & 2.63 & 3.10 \\
\hline $\mathrm{Ga}_{\mathrm{Sb}}^{2-}$ & 1.77 & 2.71 \\
\hline $\mathrm{Ga}_{\mathrm{Sb}}^{1-}$ & 1.50 & 2.44 \\
\hline $\mathrm{Ga}_{\mathrm{Sb}}^{0}$ & 1.37 & 2.30 \\
\hline $\operatorname{Sb}_{\mathrm{Ga}}^{1+}\left(T_{d}\right)$ & 1.91 & 0.97 \\
\hline $\mathrm{Sb}_{\mathrm{Ga}}^{2+}\left(T_{d}\right)$ & 1.56 & 0.63 \\
\hline $\mathrm{Sb}_{\mathrm{Ga}}^{0}\left(C_{3 v}\right)$ & 2.76 & 1.83 \\
\hline $\mathrm{Ga}_{i}^{1+}\left(T_{a}\right)$ & 1.38 & 1.85 \\
\hline $\mathrm{Ga}_{i}^{1+}\left(T_{c}\right)$ & 1.04 & 1.50 \\
\hline $\mathrm{Sb}_{i}^{1+}\left(T_{c}\right)$ & 4.14 & 3.68 \\
\hline $\mathrm{Sb}_{i}^{2+}\left(T_{c}\right)$ & 3.68 & 3.21 \\
\hline $\mathrm{Sb}_{i}^{3+}\left(T_{c}\right)$ & 3.49 & 3.02 \\
\hline $\mathrm{Sb}_{i}^{2+}\left(T_{a}\right)$ & 4.22 & 3.75 \\
\hline $\mathrm{Sb}_{i}^{3+}\left(T_{a}\right)$ & 3.99 & 3.52 \\
\hline $\mathrm{Sb}_{i}^{1+}(H)$ & 3.78 & 3.31 \\
\hline$(\mathrm{Sb}-\mathrm{Sb})_{\mathrm{Sb}\langle 110\rangle}^{1-}{ }^{\mathrm{a}}$ & 4.68 & 4.22 \\
\hline$(\mathrm{Sb}-\mathrm{Sb})_{\mathrm{Sb}\langle 110\rangle}^{0}$ & 3.97 & 3.51 \\
\hline$(\mathrm{Sb}-\mathrm{Sb})_{\mathrm{Sb}\langle 110\rangle}^{1+}$ & 3.60 & 3.13 \\
\hline
\end{tabular}

${ }^{\mathrm{a}}$ Split-interstitial rotates away from the initial $\langle 110\rangle$ orientation.

$(2-/ 3-)$ ionization level is relatively close to the conduction band minimum (CBM) due to the smaller band gap. However, the LDA calculations by Hakala et $a l .{ }^{6}$ missed the neutral charge state and the $(0 / 1-)$ ionization level close to VBM. In all the different charge states the $\mathrm{Sb}$ atoms neighboring the $\mathrm{Ga}$ vacancy relax inwards around $11 \%$ of the bond distance conserving the $T_{d}$ symmetry. This reflects the rather delocalized nature of the electrons on the Ga vacancy deep levels.

The $\mathrm{Sb}$ vacancy is stable in the charge states $+3,+1$, 0 , and -2 . In comparison to the LDA results ${ }^{6}$ the use of the HSE06 functional has stabilized the +3 state and the -2 charge state is now stable instead of the -1 charge state. For the As vacancy in GaAs the HSE06 functional gives also the charge state -2 very close to CBM, but in addition the -1 charge state is stable and the +3 charge state is missing. ${ }^{23}$ On the other hand, similar to the present result for the $\mathrm{Sb}$ vacancy semilocal functionals give the negative- $U$ transition $(3+/ 1+)$ for the As vacancy. ${ }^{11}$ The relaxation of the $\mathrm{Ga}$ atoms around the $\mathrm{Sb}$ vacancy depends strongly and rather linearly on the charge state so that the inward relaxation of $23 \%$ of the double negative vacancy vanishes for the singly positive vacancy and the relaxation for the triply positive charge state is outwards of $20 \%$. This is a result of the rather localized deep level electrons and the fact that Ga atoms are clearly smaller than the $\mathrm{Sb}$ atoms. The localized character is reflected also in the breaking of the $T_{d}$ symmetry for the neutral $\mathrm{Sb}$ vacancy.

The $\mathrm{Ga}_{\mathrm{sb}}$ antisite in $\mathrm{GaSb}$ was found to be stable in the charge states from 0 to -2 in agreement with the previous 
LDA calculations. ${ }^{6}$ For comparison, the HSE06 calculations for the $\mathrm{Ga}_{\mathrm{As}}$ antisite in GaAs give also the +1 charge state near the VBM. ${ }^{23}$ The $\mathrm{Ga}_{\mathrm{Sb}}$ antisite preserves the $T_{d}$ symmetry in all its charge states so that the relaxation is, reflecting the small size of the Ga atom, inwards and rather constant, around $7 \%$.

The $\mathrm{Sb}_{\mathrm{Ga}}$ antisite in GaSb is stable in the charge states +2 and +1 , with a symmetry-conserving outward relaxation of around $7 \%$ of the nearest-neighbor $\mathrm{Sb}$ atoms. The existence of the positive charge states is in a striking contrast with the previous LDA calculations which predicted the neutral state as the only stable charge state. ${ }^{6}$ The position of the ionization level $(2+/ 1+)$ located at $0.36 \mathrm{eV}$ [i.e., near the middle of the gap resembles the behavior of the HSE06 results for the $\mathrm{As}_{\mathrm{Ga}}$ antisite in GaAs (Refs. 10,23)]. The HSE06 calculations for GaAs indicate also that the neutral $\mathrm{As}_{\mathrm{Ga}}$ antisite is stable in the uppermost third of the band gap. Similar results were also found for $\mathrm{Sb}_{\mathrm{Al}}$ antisite in AlSb in Ref. 12, were charge states from +2 to -1 were found to be stable. We also performed LDA calculations for the $\mathrm{Sb}_{\mathrm{Ga}}$ antisite, with the same system settings (system size and energy correction term) as with the HSE06 functional. Our LDA results for $\mathrm{Sb}_{\mathrm{Ga}}$ antisite resembles those of Ref. 6 , except that now the +1 charge state is stable with Fermi levels near the valence band edge.

In accordance with semilocal functional calculations for III-V compound semiconductors ${ }^{11,12,24}$ our HSE06 calculations predict the existence of a metastable $C_{3 v}$ configuration for the neutral $\mathrm{Sb}_{\mathrm{Ga}}$ antisite (in GaAs this corresponds to the metastability of the EL2 defect). In the $C_{3 v}$ configuration the $\mathrm{Sb}$ atom from the center of the antisite defect has moved $1.56 \AA$ along the [111] direction and penetrated the triangle formed by the three neighboring $\mathrm{Sb}$ atoms. The metastable $C_{3 v}$ configuration has only the neutral charge state in the band gap. We have calculated the energy landscape of the neutral $\mathrm{Sb}_{\mathrm{Ga}}^{0}$ antisite with respect to the movement of the central $\mathrm{Sb}$ atom along the [111] direction towards the open interstitial position by using the climbing-image nudged elastic band method. ${ }^{25}$ The results are shown in Fig. 2. The HSE06 functional calculation gives the metastable $C_{3 v}$ configuration at the same energy as the neutral $T_{d}$ configuration. The thermal barrier from the $T_{d}$ configuration to the $C_{3 v}$ configuration is $0.25 \mathrm{eV}$ which would allow a high jumping rate already at the room temperature.

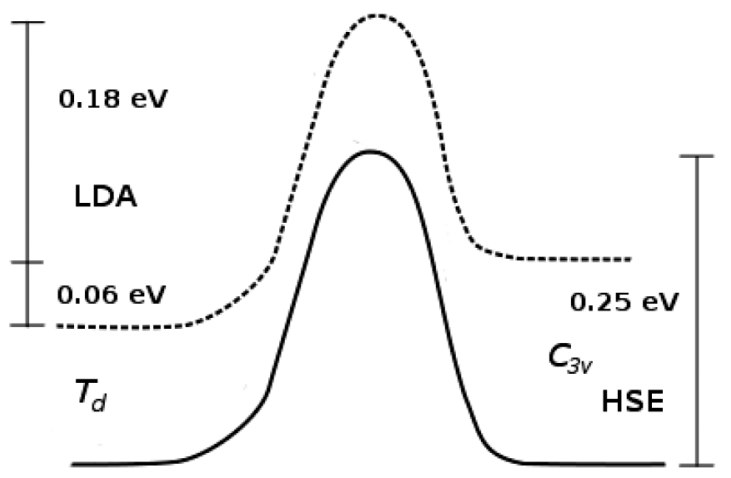

FIG. 2. Schematic illustration of the transformation from the $T_{d}$ configuration to the $C_{3 v}$ configuration for the neutral $\mathrm{Sb}_{\mathrm{Ga}}$ antisite defect. The results of the HSE06 (solid line) and LDA (dashed line) functional calculations are shown.
This differs from the situation for the LDA functional where the $T_{d}$ configuration is lower in energy by $0.06 \mathrm{eV}$ and the barrier from the $C_{3 v}$ configuration back to $T_{d}$ configuration is only $0.18 \mathrm{eV}$.

The Ga interstitial is stable only in the +1 charge state and in the tetrahedral configurations $T_{c}$ and $T_{a}$. In both configurations there is a symmetry-conserving 5\% outwards relaxation. The $\mathrm{Sb}$ interstitial exist in all the sites $T_{c}, T_{a}$, and $H$ having the stable charge states from +3 to +1 at the $T_{c}$ site, +2 and +1 at the $T_{a}$ site, and the charge state +1 at the $H$ site. The LDA results by Hakala et al. ${ }^{6}$ are roughly similar finding the singly positive $\mathrm{Ga}$ interstitial in the $T_{c}$ site, the triply and singly positive $\mathrm{Sb}$ interstitials in the $T_{c}$ site, and the singly positive $\mathrm{Sb}$ interstitial in the $H$ site. The semilocal functional results for GaAs indicate that also the doubly and triply positive $\mathrm{Ga}$ interstitials are stable. ${ }^{11}$ Moreover, those calculations $^{11}$ predicted the existence of the singly negative As interstitial in the upper part of the band gap and, parallel to our results for the $\mathrm{Sb}$ interstitial, the appearance of the $(3+/ 1+)$ ionization level.

The Sb split-interstitial is stable in the $+1,0$, and -1 charge states. In the +1 and 0 charge states the $\mathrm{Sb}$ split-interstitial remains oriented along the initial $\langle 110\rangle$ direction, but in the -1 charge state it rotates away from it. This is in contrast to $\mathrm{AlSb}$ were the $(\mathrm{Sb}-\mathrm{Sb})_{\mathrm{Sb}\langle 110\rangle}$ split-interstitial was found to be stable in all the charge states from +2 to -2 (Ref. 19) and remain oriented along the $\langle 110\rangle$ direction in all charge states. According to the Table I the formation energy of the $(\mathrm{Sb}-\mathrm{Sb})_{\mathrm{Sb}\langle 110\rangle}$ split-interstitial is also rather high in $\mathrm{GaSb}$.

\section{B. Formation energies of native point defects in different growth conditions}

Figures 3(a) and 3(b) give the formation energies for most important point defects in $\mathrm{GaSb}$ as a function of the Fermi level in Ga-rich and Sb-rich growth conditions, respectively. The figures are based on the numerical data in Table I. The formation energies of the $\mathrm{Sb}$ interstitials and $(\mathrm{Sb}-\mathrm{Sb})_{\mathrm{Sb}\langle 110\rangle}$ split-interstitials are considerably high when compared to the other defects and, therefore, as thermodynamically unlike defects they are not shown.

In the Ga-rich growth conditions the defects with the lowest formation energies are the $\mathrm{Ga}_{i}^{1+}\left(T_{c}\right)$ interstitial and the $\mathrm{Ga}_{\mathrm{Sb}}^{2-}$ antisite. Moving to the Sb-rich growth conditions turns the $\mathrm{Sb}_{\mathrm{Ga}}$ antisite into the most favorable defect with the exception of the region very close to the CBM. Moreover, the formation energy of the Ga vacancy decreases and that of the $\mathrm{Ga}_{i}^{1+}\left(T_{c}\right)$ interstitial increases.

In general our HSE06 functional results show characteristics similar to those by Hakala et al ${ }^{6}$ obtained by using the LDA although the present formation energies are clearly larger. For example, the formation energies of $\mathrm{Ga}$ and $\mathrm{Sb}$ vacancies have raised by roughly $1 \mathrm{eV}$. The main qualitative difference with respect to the previous LDA results ${ }^{6}$ is, however, that hybrid functionals favor more positive charge states. This trend was found also in the case of oxygen vacancies in transparent conducting oxides by Ágoston et al. ${ }^{26}$ and it reflects mainly the relative lowering of the valence band states due to the reduction in the spurious electron self-interaction. Most notably, this makes the positive charge states of the $\mathrm{Sb}_{\mathrm{Ga}}$ antisite stable and 



FIG. 3. (Color online) Formation energies for stable charge states of point defects in GaSb as a function of the Fermi level in (a) Ga-rich and (b) Sb-rich growth conditions.

causes the appearance of the donor level $(2+/ 1+)$ in the band gap at $0.36 \mathrm{eV}$ above the VBM.

We have also performed most of the calculations using LDA with the same system settings as with the HSE06 functional. The LDA results are rather similar to the results in Ref. 6 and thus the qualitative differences between our HSE06 results and those of Ref. 6 cannot be explained by differences in computational details, but reflect the differences between the different functionals used.

\section{Extrinsic point defects $\mathrm{C}_{\mathrm{Sb}}$ and $\mathrm{O}\left(T_{c}\right)$}

Figure 4 shows the formation energies for substitutional carbon $\mathrm{C}_{\mathrm{Sb}}$ and interstitial oxygen $\mathrm{O}\left(T_{c}\right)$ as a function of the Fermi level. $\mathrm{C}_{\mathrm{Sb}}$ is in the singly negative charge state for the whole range of the Fermi level similarly to AlSb (Ref. 13). The $\mathrm{C}_{\mathrm{Sb}}$ remains in the $T_{d}$ symmetry during relaxation. Interstitial oxygen in the $T_{c}$ site has stable charge states -1

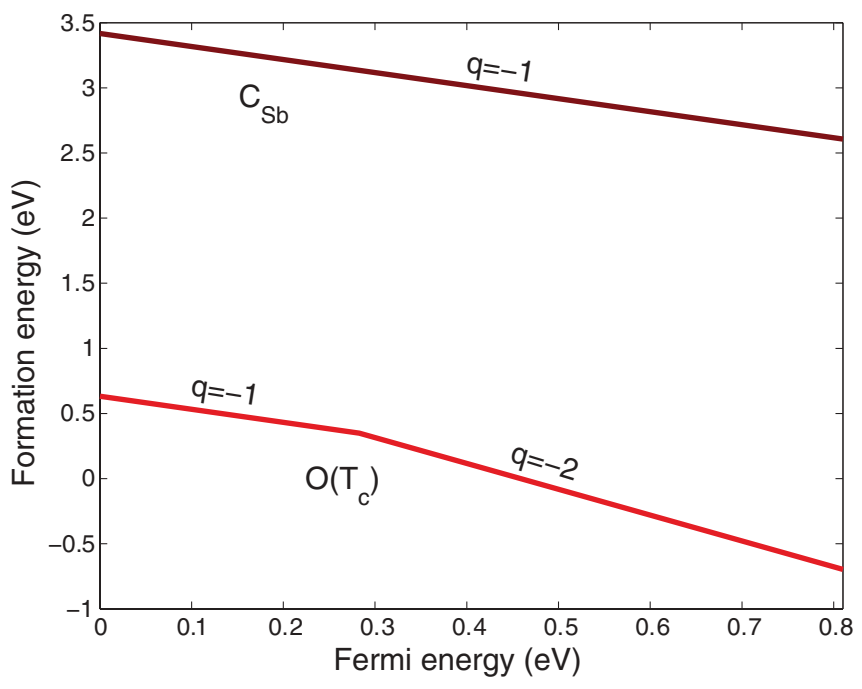

FIG. 4. (Color online) Formation energies for extrinsic impurity defects $\mathrm{C}_{\mathrm{Sb}}$ and $\mathrm{O}\left(T_{c}\right)$ as a function of the Fermi level in Sb-rich growth conditions. and -2 in the gap and the ionization level $(-1 /-2)$ is located at $0.29 \mathrm{eV}$. The $\mathrm{O}\left(T_{c}\right)$ has the $C_{3 v}$ symmetry at the -1 charge state and $T_{d}$ symmetry at the -2 charge state. Both $\mathrm{C}_{\mathrm{Sb}}$ and $\mathrm{O}\left(T_{c}\right)$ act as acceptors for the whole range of the Fermi level and can thus trap excess electrons and contribute to the $p$-type conductivity. According to Fig. 4 the formation energy of the $\mathrm{C}_{\mathrm{Sb}}$ is very high compared to that of $\mathrm{O}\left(T_{c}\right)$ and thus the oxygen would clearly be a much more likely candidate to compensate the possible donor character of the $\mathrm{Sb}_{\mathrm{Ga}}$ antisite.

\section{DISCUSSION: $p$-TYPE CONDUCTIVITY OF UNDOPED GaSb}

The very reasonable qualitative agreement of our HSE06 functional results with the previous semilocal functional results for defects in GaSb and GaAs and with the HSE06 results for defects in GaAs gives credence to our discussion about the origin of the $p$-type conductivity of GaSb.

According to Fig. 3, the Gasb antisite acts as a single and as a double acceptor for Fermi levels above 0.13 and $0.27 \mathrm{eV}$, respectively. Thus, in Ga-rich growth conditions the most likely source of $p$-type conductivity are the $\mathrm{Ga}_{\mathrm{Sb}}^{2-}$ antisites. Compensation due to the $\mathrm{Ga}_{i}^{1+}\left(T_{c}\right)$ interstitials is not expected because of their high mobility and recombination with open volume defects already below the room temperature. When going from $\mathrm{Ga}$-rich to $\mathrm{Sb}$-rich growth conditions the formation energy of the $\mathrm{Ga}_{\mathrm{Sb}}$ antisite increases considerably predicting a decrease in the $p$-type conductivity. This has actually been observed in experiments ${ }^{27}$ where a significant drop in the number of background acceptors was observed.

Especially in Sb-rich growth conditions the formation energy of the $\mathrm{Sb}_{\mathrm{Ga}}$ antisite is notably low. It is a single or even a double donor over the whole range of the Fermi level in the band gap and its existence would make $\mathrm{GaSb}$ $n$-type when the growth stoichiometry turns to the Sb-rich side. One possible explanation to this apparent discrepancy could be the turning of the $T_{d}$ configuration of the $\mathrm{Sb}_{\mathrm{Ga}}$ antisite to the $C_{3 v}$ configuration. The latter configuration is neutral for all the Fermi level positions. Given the accuracy of our calculations as reflected also in the magnitude and 


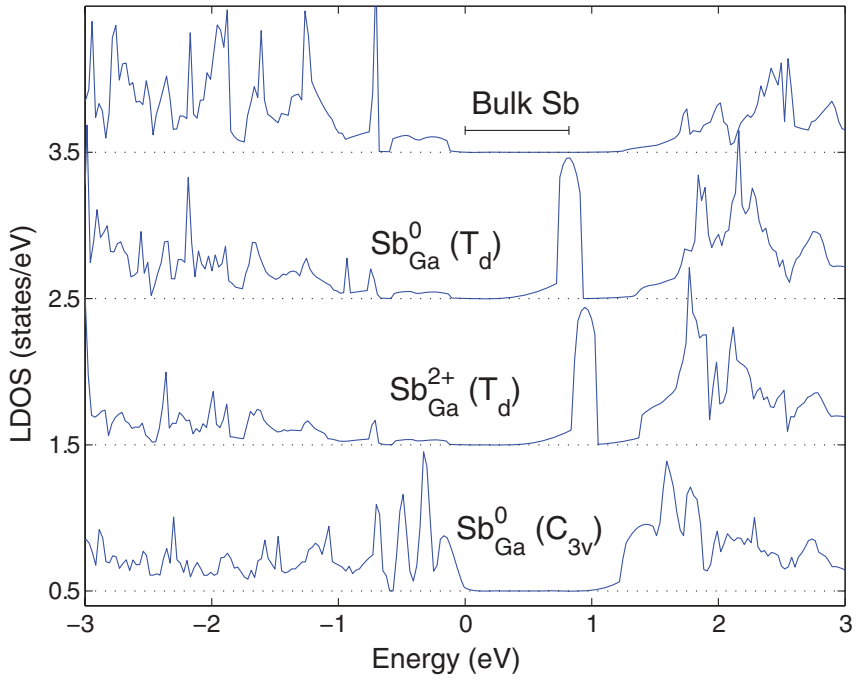

FIG. 5. (Color online) LDOS for the Sb atom in bulk GaSb and in the defect systems. The LDOS shown for the defect systems correspond to the defect atom. The energy zero coincides with the top of the valence band at the $\Gamma$ point. The original zero LDOS values of the adjacent curves given by horizontal dashed lines are shifted relative to each other. The solid segment at the topmost LDOS curve indicates the band gap in bulk GaSb.

trend in the difference between the LDA and HSE06 results in Fig. 2 above, the actual situation could be that the neutral $C_{3 v}$ configuration is energetically favored and easily reachable by thermal excitation from the neutral $T_{d}$ configuration. The neutral $T_{d}$ configuration could result when the strong and narrow resonance at the CBM seen in the LDOS of the $\mathrm{Sb}_{\mathrm{Ga}}^{2+}$ in Fig. 5 for the $T_{d}$ configuration captures electrons from the conduction band.

In addition to the above over-the-thermal-barrier route the $C_{3 v}$ configuration could be also reached from the neutral $T_{d}$ configuration by photoexcitation according to the model proposed by Dabrowski and Scheffler ${ }^{28}$ for the EL2 defect in GaAs. In this model a resonance level in the conduction band induced by the $\mathrm{As}_{\mathrm{Ga}}$ antisite is occupied due to (photo) excitation from the deep level in the band gap and then the defect relaxes spontaneously to the metastable $C_{3 v}$ configuration. Indeed the LDOS in Fig. 5 shows that also the $\mathrm{Sb}_{\mathrm{Ga}}$ antisite induces rather localized states at about $1.8 \mathrm{eV}$ above the VBM. The strong resonance just above the CBM, which was discussed above, corresponds to the deep gap state of the EL2 defect in GaAs. According to partial charge densities the character of the resonance states at about $1.8 \mathrm{eV}$ agree with the Dabrowski-Scheffler model. Moreover, as shown in Fig 5, in the $C_{3 v}$ configuration there are resonance states just below the VBM. These correspond to the level found by Dabrowski and Scheffler in the band gap just above the VBM. Thus, we propose the model that electrons excited to the conduction band are captured effectively by the resonance induced by the positive antisite close to the CBM. A further photoexcitation of the captured electrons to the resonance states at about $1.8 \mathrm{eV}$ above the VBM results in the relaxation to the $C_{3 v}$ configuration in accordance with the Dabrowski-Scheffler model. The resonances induced by the neutral $C_{3 v}$ defect are relatively far from the band gap and thus less localized than those induced by the $\mathrm{Sb}$ antisite and therefore they are expected to be less active in electron capture of photoexcitation processes.

Another explanation to $p$-type conductivity in the Sb-rich growth conditions are the extrinsic point defects, namely, carbon in the substitutional $\mathrm{Sb}$ site and oxygen in the interstitial cation site. Indeed, according to our calculations the formation energy for the oxygen interstitial is very low. These defects act as acceptors and could compensate the donor character of the $\mathrm{Sb}_{\mathrm{Ga}}$ antisite. However, to our knowledge undoped $\mathrm{GaSb}$ is always $p$-type regardless of the method of the growth suggesting that the extrinsic defects are not crucial for the $p$-type conductivity of GaSb.

\section{CONCLUSION}

We have studied native point defects in GaSb within the DFT framework. The use of the hybrid exchange-correlation functional was found to affect crucially the important relative energetics of the different defects. The lowest-energy defects found are the $\mathrm{Ga}$ antisite, the $\mathrm{Ga}$ interstitials, and the $\mathrm{Sb}$ antisite. Especially, the formation energy of the $\mathrm{Ga}_{\mathrm{Sb}}^{2-}$ antisite was predicted to be considerably low in Ga-rich growth conditions and thus the source of the $p$-type conductivity. However, in contrast to earlier LDA studies also the formation energy of the $\mathrm{Sb}$ antisite was found to be considerably low in Sb-rich growth conditions. This is due to the positive charge states stabilized by the hybrid functional. The existence of the positive charge states means also that the $\mathrm{Sb}$ antisite should act as a donor in Sb-rich growth conditions contributing to $n$-type conductivity (or the efficient compensation of the $p$-type conductivity), which is not observed in the experiments. As a solution to this discrepancy we studied the metastability of the $\mathrm{Sb}$ antisite. In addition we found that extrinsic point defects $\mathrm{C}_{\mathrm{Sb}}$ and $\mathrm{O}\left(T_{c}\right)$ act as acceptors in $\mathrm{GaSb}$ and especially $\mathrm{O}\left(T_{c}\right)$, having a low formation energy, might compensate the electrical activity of the $\mathrm{Sb}_{\mathrm{Ga}}$ antisite.

\section{ACKNOWLEDGMENTS}

This work has been supported by the Academy of Finland through the Center of Excellence program. The computer time was provided by the Finnish IT Center for Science and Technology (CSC).
${ }^{1}$ P. S. Dutta, H. L. Bhat, and V. Kumar, J. Appl. Phys. 81, 5821 (1997).

${ }^{2}$ J. Paajaste, S. Suomalainen, R. Koskinen, A. Härkönen, M. Guina, and M. Pessa, J. Cryst. Growth 311, 1917 (2009).
${ }^{3}$ D. Wang, S. P. Svensson, L. Shterengas, G. Belenky, C. S. Kim, I. Vurgaftman, and J. R. Meyer, J. Appl. Phys. 105, 014904 (2009). ${ }^{4}$ V. Virkkala, V. Havu, F. Tuomisto, and M. J. Puska, Phys. Rev. B 85, 085134 (2012). 
${ }^{5}$ C. C. Ling, M. K. Lui, S. K. Ma, X. D. Chen, S. Fung, and C. D. Beling, Appl. Phys. Lett. 85, 384 (2004).

${ }^{6}$ M. Hakala, M. J. Puska, and R. M. Nieminen, J. Appl. Phys. 91, 4988 (2002).

${ }^{7}$ A. Peles, A. Janotti, and C. G. Van de Walle, Phys. Rev. B 78, 035204 (2008).

${ }^{8}$ R. M. Nieminen, Modell. Simul. Mater. Sci. Eng. 17, 084001 (2009).

${ }^{9}$ C. Freysoldt, J. Neugebauer, and C. G. Van de Walle, Phys. Status Solidi B 248, 1067 (2010).

${ }^{10}$ H.-P. Komsa and A. Pasquarello, Phys. Rev. B 84, 075207 (2011).

${ }^{11}$ P. A. Schultz and O. A. von Lilienfeld, Modell. Simul. Mater. Sci. Eng. 17, 084007 (2009).

${ }^{12}$ M.-H. Du, Phys. Rev. B 79, 045207 (2009).

${ }^{13}$ P. Erhart, D. Åberg, and V. Lordi, Phys. Rev. B 81, 195216 (2010).

${ }^{14}$ G. Kresse and J. Furthmüller, Comput. Mater. Sci. 6, 15 (1996).

${ }^{15}$ G. Kresse and D. Joubert, Phys. Rev. B 59, 1758 (1999).

${ }^{16}$ J. Heyd, J. E. Peralta, G. E. Scuseria, and R. L. Martin, J. Chem. Phys. 123, 174101 (2005).

${ }^{17}$ L. S. dos Santos, W. G. Schmidt, and E. Rauls, Phys. Rev. B 84, 115201 (2011).
${ }^{18}$ I. Vurgaftman, J. R. Meyer, and L. R. Ram-Mohan, J. Appl. Phys. 89, 5815 (2001).

${ }^{19}$ D. Åberg, P. Erhart, A. J. Williamson, and V. Lordi, Phys. Rev. B 77, 165206 (2008).

${ }^{20}$ Ternary and Quaternary $A_{3} B_{5}$ Semiconductors in Handbook Series on Semiconductor Parameters, edited by M. Levinshtein, S. Rumyantsev, and M. Shur (World Scientific, Singapore, 1999), Vol. 2.

${ }^{21}$ C. G. Van de Walle and J. Neugebauer, J. Appl. Phys. 95, 3851 (2004).

${ }^{22}$ D. D. Wagman, W. H. Evans, V. B. Parker, R. H. Schumm, I. Halow, S. M. Bailey, K. L. Churney, and R. L. Nuttal, J. Phys. Chem. Ref. Data 11, Supplement 2 (1982).

${ }^{23}$ H.-P. Komsa and A. Pasquarello, J. Phys.: Condens. Matter 24, 045801 (2012)

${ }^{24}$ M. J. Caldas, J. Dabrowski, A. Fazzio, and M. Scheffler, Phys. Rev. Lett. 65, 2046 (1990).

${ }^{25}$ G. Henkelman and H. Jónsson, J. Chem. Phys. 113, 9978 (2000).

${ }^{26}$ P. Ágoston, K. Albe, R. M. Nieminen, and M. J. Puska, Phys. Rev. Lett. 103, 245501 (2009).

${ }^{27}$ Y. J. Van der Meulen, J. Phys. Chem. Solids 28, 25 (1967).

${ }^{28}$ J. Dabrowski and M. Scheffler, Phys. Rev. Lett. 60, 2183 (1988). 